\title{
Antitumor activity of vorinostat-incorporated nanoparticles against human cholangiocarcinoma cells
}

\author{
Tae Won Kwak ${ }^{1 \dagger}$, Do Hyung Kim ${ }^{2 \dagger}$, Young-II Jeong ${ }^{1 *}$ and Dae Hwan Kang ${ }^{1,3^{*}}$
}

\begin{abstract}
Background: The aim of this study is to evaluate the anticancer activity of vorinostat-incorporated nanoparticles (vorinostat-NPs) against HuCC-T1 human cholangiocarcinoma cells. Vorinostat-NPs were fabricated by a nanoprecipitation method using poly(DL-lactide-co-glycolide)/poly(ethylene glycol) copolymer.

Results: Vorinostat-NPs exhibited spherical shapes with sizes $<100 \mathrm{~nm}$. Vorinostat-NPs have anticancer activity similar to that of vorinostat in vitro. Vorinostat-NPs as well as vorinostat itself increased acetylation of histone-H3. Furthermore, vorinostat-NPs have similar effectiveness in the suppression or expression of histone deacetylase, mutant type p53, p21, and PARP/cleaved caspase-3. However, vorinostat-NPs showed improved antitumor activity against HuCCT1 cancer cell-bearing mice compared to vorinostat, whereas empty nanoparticles had no effect on tumor growth. Furthermore, vorinostat-NPs increased the expression of acetylated histone $\mathrm{H} 3$ in tumor tissue and suppressed histone deacetylase (HDAC) expression in vivo. The improved antitumor activity of vorinostat-NPs can be explained by molecular imaging studies using near-infrared (NIR) dye-incorporated nanoparticles, i.e. NIR-dye-incorporated nanoparticles were intensively accumulated in the tumor region rather than normal one.
\end{abstract}

Conclusions: Our results demonstrate that vorinostat and vorinostat-NPs exert anticancer activity against HuCC-T1 cholangiocarcinoma cells by specific inhibition of HDAC expression. Thus, we suggest that vorinostat-NPs are a promising candidate for anticancer chemotherapy in cholangiocarcinoma.

Keywords: Vorinostat, Cholangiocarcinoma, Nanoparticles, Poly(DL-lactide-co-glycolide), Block copolymer, Cancer chemotherapy, Drug targeting

\section{Background}

Vorinostat (suberoylanilide hydroxamic acid, SAHA) is known to one of histone deacetylase inhibitor (HDACi) [1]. In acetylation process, acetyl group in one molecule is transferred to another and deacetylation reaction is to remove acetyl group from a molecule. HDAC has an important role in the transcriptional regulation through stabilization of DNA-histone interaction and deacetylation process is known to have relationship with carcinogenesis [1]. HDACis such as vorinostat act as a chelator

\footnotetext{
*Correspondence: nanomed@naver.com; sulsulpul@naver.com †Tae Won Kwak and Do Hyung Kim equally contributed to this work 1 Biomedical Research Institute, Pusan National University Hospital, 179 Gudeok-ro, Seo-gu, Busan 602-739, Republic of Korea Full list of author information is available at the end of the article
}

for zinc ions in the active site of histone deacetylases (HDACs) and vorinostat is regarded as a promising cancer chemotherapeutic drug [1]. Vorinostat has been approved by the FDA for treatment of cutaneous $\mathrm{T}$ cell lymphoma [1, 2]. Accumulation of acetylated histones and acetylated proteins has correlation with $p 21^{W A F 1}$ gene expression, apoptotic signals such as mutant-type $p 53$ and active-type caspase expression, cell differentiation and cell death [1-5]. In recent clinical trials, the safety and anticancer efficacy vorinostat has been evaluated against gastrointestinal (GI) cancer patient [3]. In the results of these trials, the report suggested that vorinostat can be used as an effective anticancer agent for GI cancer [3]. Vorinostat induced both apoptosis and autophagy in gastric cancer cell lines and has shown clinical benefits 
for gastric cancer patients [6, 7]. The anticancer activity of vorinostat has also investigated against colon cancer, glioma, lung cancer, breast cancer and hepatocellular carcinoma in preclinical or clinical trials, both as a single treatment or combination with other types of anticancer drugs [5-8]. We previously reported that vorinostat exhibits anticancer efficacy against $\mathrm{HuCC}-\mathrm{T} 1$ human cholangiocarcinoma (CCA) cells [9]. In this report, we show that vorinostat is involved in growth inhibition, apoptosis of $\mathrm{HuCC}-\mathrm{T} 1$ cells in vitro and anti-tumor activity of HuCC-T1 cell-bearing xenograft model in vivo.

CCA is a malignant tumor that occurs in the epithelium of the biliary tract [10]. Although the rate of incidence of CCA has increased worldwide, the reason for its increase remains unclear $[11,12]$. Current treatment options for CCA include surgical resection, radiotherapy, chemotherapy, stent displacement and immunotherapy [13-15]. Although surgical resection is believed to be a curative treatment option for CCA, patients with CCA are frequently diagnosed at an unresectable stage [16]. Chemotherapeutic approaches for CCA are considered to increase patient survival and quality of life [12]. Various chemotherapeutic agents such as gemcitabine, cisplatin, oxaliplatin, capecitabine and 5-fluorouracil have been tested as single agents or in combination in clinical trials for CCA $[17,18]$. Even though the combination of some anticancer agents have been reported to have therapeutic advantages, systemic chemotherapy using conventional anticancer agents is still ineffective and shows an insignificant increase in survival period. In fact, current standard chemotherapeutic treatment for CCA patients is normally gemcitabine plus cisplatin [18, 19]. Even though combination of these chemotherapeutic agents delayed onset of progression, most cases still succumbed to CCA and has no significant advances in survivability [20]. Because most of chemotherapeutic agents showed minimal survival gain and chemotherapeutic agents have difficulties in delivery to CCA, targeted therapy for CCA patients has been proposed [21]. Novel treatment options for a chemotherapeutic approach for CCA are required to improve patient survivability.

Nanomedicine such as nanoparticles, liposomes and polymeric micelles have advantages in targeting malignant solid tumor because they have small sizes of $<1000 \mathrm{~nm}$ and unique structures that can amplify the anticancer activity of conventional drugs [22-27]. In recent decades, nanomedicine-based drug delivery systems have also been investigated to target CCA cells for diagnosis and chemotherapeutic treatment [22-27]. Magnetic nanoparticles were reported to be a useful device for the diagnosis of intrahepatic CCA [22, 23]. Magnetic drug nanoparticles enveloping chemotherapeutic drugs were reported to be an effective treatment for the inhibition of CCA cell proliferation in a tumor xenograft model of nude mice [24]. Totawa et al. reported that hybrid liposomes were specifically accumulated in human CCA cells and induced cell cycle arrest [25]. In our previous study, chitosan nanoparticles incorporating all-trans retinoic acid were demonstrated to be effective in inhibiting the invasion, migration and proliferation of human CCA cells [26]. Stimuli-sensitive nanoparticles can also be considered to target CCA cells [27].

In this study, we prepared vorinostat-NPs using biodegradable polymers to assess their anticancer effects on HuCC-T1 cells in vitro and in vivo. The efficacy of vorinostat and vorinostat-NPs in HuCC-T1 cells was studied using western blotting, immunohistochemistry and a HuCC-T1 xenograft model in nude mice.

\section{Results}

Characterization of vorinostat-incorporated nanoparticles

Vorinostat-incorporated nanoparticles (vorinostatNPs) were fabricated using the nanoprecipitation method. Vorinostat and poly(DL-lactide-co-glycolide)/ poly(ethylene glycol) (LGE) block copolymer was dissolved in organic solvent. Then this solution was poured into aqueous solution and the organic solvent was removed. At these procedures, there is no apparent precipitation of vorinostat in the aqueous phase. To remove remained organic solvents and emulsifier (pluronic F68), vorinostat-NPs were separated by centrifugation and washing process. As shown in Table 1, experimental drug loading of vorinostat into the nanoparticles was slightly lower than the theoretical value. This might be due to the liberation of vorinostat from the nanoparticles during the preparation procedure, thereby causing a decrease in the effective drug content. Figure 1 shows the characteristics of vorinostat-NPs. X-ray powder diffractograms (XRD) measurement of lyophilized nanoparticles was employed to confirm whether or not free drug was remained in the nanoparticle solution as shown in Fig. 1a. As shown in Fig. 1a, vorinostat alone has sharp crystalline peaks while empty nanoparticles (empty-NPs) have broad peak properties. Interestingly, vorinostat-NPs also have broad peak properties as similar to empty-NPs whereas the physical

Table 1 Evaluation of drug contents of vorinostat-NPs

\begin{tabular}{|c|c|c|c|}
\hline & \multirow{2}{*}{$\begin{array}{l}\text { Polymer/vorinostat } \\
\text { weight ratio }(\mathrm{mg} / \mathrm{mg})\end{array}$} & \multicolumn{2}{|c|}{ Drug contents $(\%, w / w)$} \\
\hline & & Theoretical & $\begin{array}{l}\text { Experimen- } \\
\text { tal }\end{array}$ \\
\hline Empty & $100 / 0$ & - & - \\
\hline Vorinostat-NP10 & $100 / 10$ & 9.1 & 8.3 \\
\hline Vorinostat-NP20 & $100 / 20$ & 16.7 & 15.1 \\
\hline
\end{tabular}

Vorinostat contents in the nanoparticles are listed 


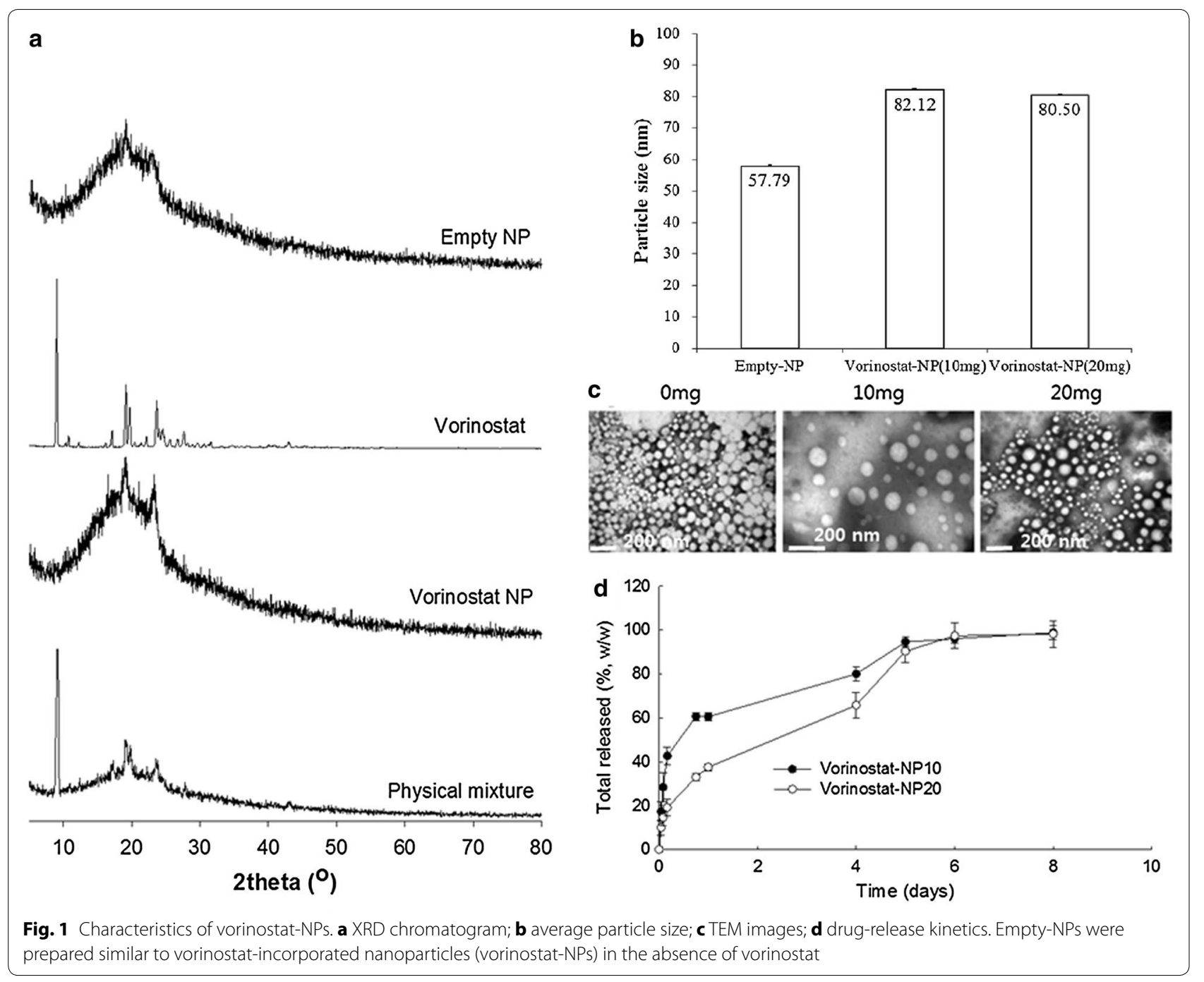

mixture of empty-NPs and vorinostat has both sharp and broad crystalline peaks. These results indicated that the intrinsic crystallinity of vorinostat was decreased by incorporation into the polymer nanoparticles and that vorinostat was molecularly distributed in the nanoparticle matrix. Furthermore, these results demonstrated that no extensive precipitation of drug has been occurred during fabrication procedure and then free drug remained was minimized. Additionally, these results also indicated that vorinostat was properly payloaded into the nanoparticles.

The average particle size was measured to investigate colloidal properties of vorinostat-NPs as shown in Fig. 1b. Particles size of nanoparticles was slightly increased when vorinostat was incorporated into the nanoparticles (Fig. 1b), but the vorinostat content of the nanoparticles did not significantly affect the change in particle diameter. As shown in Fig. 1c, empty-NPs and vorinostat-NPs have spherical shapes under transmission electron microscopy (TEM) observation with small particle sizes of $<200 \mathrm{~nm}$. Figure $1 \mathrm{~d}$ shows the release properties characteristics of vorinostat from the nanoparticles. The initial burst release of the drug was observed for 1 day and, subsequently, vorinostat-NPs revealed sustained behavior over 5 days. In particular, the release rate of vorinostat was slower when the vorinostat content in the nanoparticles was higher. These phenomena might be due to that hydrophobic drugs in the nanoparticles can be aggregated at higher drug content and then aggregated drug released slowly due to the limited solubility in the aqueous phase. Gref et al. reported that increased drug contents of hydrophobic drugs into core-shell type nanospheres lead to aggregation or crystallization in the cores of the nanospheres [28]. The crystallization 
or aggregation of hydrophobic drug in the core of the nanospheres led slow rate of dissolution and diffusion of drugs into the aqueous phase [28]. Then, the release rate of hydrophobic should be slower at higher drug loading than lower drug loading.

\section{In vitro anticancer activity of vorinostat-incorporated nanoparticles}

Figure 2 shows the anticancer activity of vorinostat and vorinostat-NPs against HuCC-T1 cells. For cytotoxicity index, viability of cells was checked in the serum-free media as shown in Fig. 2a. The viability of the cells was slightly decreased according to the increase of vorinostat concentration. In particular, vorinostat-NPs has higher cytotoxicity than vorinostat alone even though empty nanoparticles have small effect on the viability of cells. In growth inhibition study, both vorinostat and vorinostatincorporated nanoparticles have low inhibitory effects on the growth of HuCC-T1 cells as shown in Fig. $2 \mathrm{~b}$. These results were compared at vorinostat concentration of 1 and $5 \mu \mathrm{g} / \mathrm{ml}$ as shown in Fig. 2c. Both vorinostat and vorinostat nanoparticles has small inhibitory effects on the cell growth at 1 and $5 \mu \mathrm{g} / \mathrm{ml}$ while empty nanoparticles did not affect to the growth of HuCC-T1 cells. Figure $2 \mathrm{~d}$ shows the apoptosis/necrosis analysis of
HuCC-T1 cells upon treatment with vorinostat and vorinostat-incorporated nanoparticles. As shown in Fig. 2d, e, the apoptosis and necrosis of $\mathrm{HuCC}-\mathrm{T} 1$ cells were significantly increased at $5 \mu \mathrm{g} / \mathrm{ml}$ of a vorinostat concentration when vorinostat or vorinostat-incorporated nanoparticles were treated. The empty nanoparticles showed minimal effect to cells.

Figure 3 shows the western blot analysis of molecular signals in HuCC-T1 cells upon treatment with vorinostat and vorinostat-NPs. As shown in Fig. 3a, acetylated histone $\mathrm{H} 3$ was evidently increased upon treatment with $5 \mu \mathrm{g} / \mathrm{ml}$ vorinostat and vorinostat-NPs, whereas emptyNPs have no effect on this signal. As shown in Fig. 3b, quantitative analysis showed a significant increase in ac-histone $\mathrm{H} 3$ expression in $\mathrm{HuCC}-\mathrm{T} 1$ cells, indicating that vorinostat-incorporated nanoparticles were effective in acetylation of Histone $\mathrm{H} 3$ as well as vorinostat itself. Furthermore, treatment of vorinostat-incorporated nanoparticles were also effective in decreasing expression of HDAC 1 and HDAC 3 as well as vorinostat itself even though HDAC2 expression was almost similar or slightly higher than control. In fact, HDAC2 and 3 expression of vorinostat-incorporated nanoparticles were slightly higher than vorinostat itself at $5 \mu \mathrm{g} / \mathrm{ml}$ concentration (Fig. 3a, c). These results might be due to the sustained

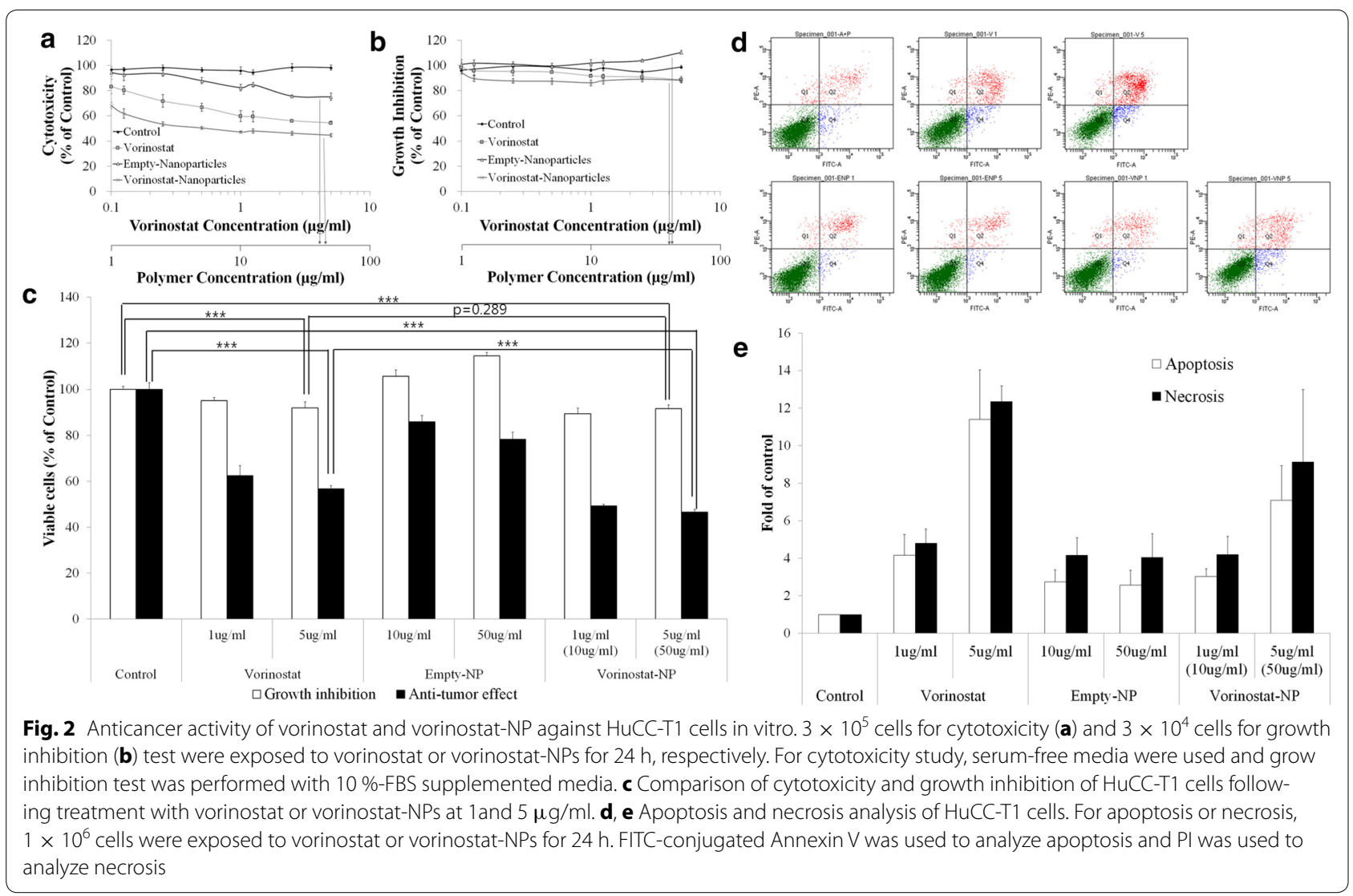




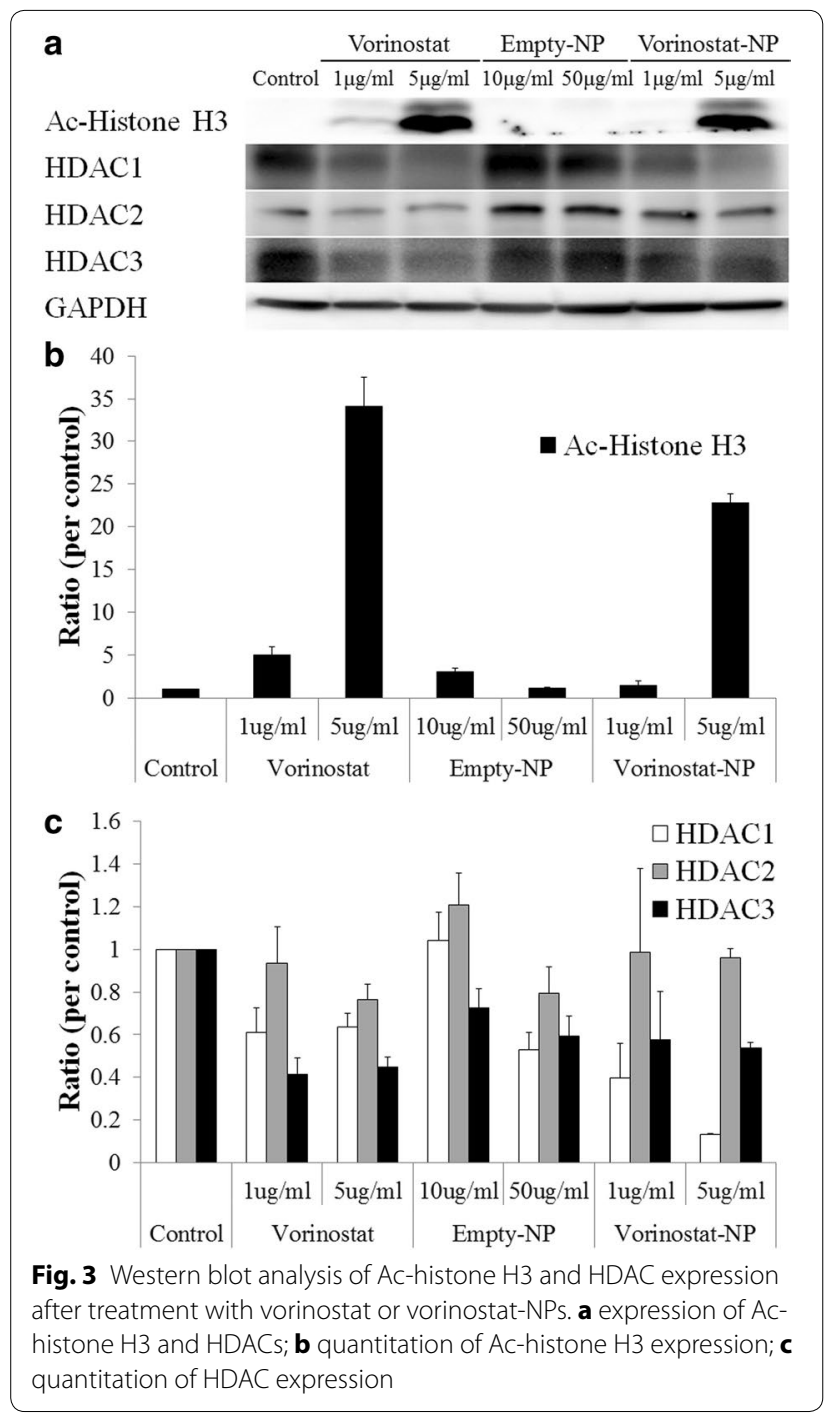

release properties of nanoparticles, i.e. nanoparticles slowly released the drug into the cell culture medium and lower concentration of intact vorinostat can affected to the cellular expression of HDAC2 and 3. Interestingly, empty-NPs affected HDAC expression in cancer cells at high concentrations $(50 \mu \mathrm{g} / \mathrm{ml})$. Because the reason for these results is not clear, further investigations are required in the future. One possible explanation of these results is that high concentrations of empty-NPs show some cytotoxic effects in the in vitro cell culture environment, even though LGE block copolymer has already been approved as a biocompatible polymer and approved for human use by the US FDA [29].

Figure 4A shows that the level of mutant p53 was significantly decreased both by vorinostat and vorinostat-NPs, whereas that of wild-type p53 was not significantly changed. The expression of p21 was significantly increased upon treatment with both vorinostat and vorinostat-NPs. Upon immunocytochemical staining of HuCC-T1 cells, a decrease in mutant-type p53 and an increase in p21 were also observed. Expression of each protein was normalized to the GAPDH as cytosolic control and lamin B as nuclear control. Figure 4B shows apoptosis signals in HuCC-T1 cells. As shown in Fig. 4B, a decrease in the level of poly-ADP ribose polymerase (PARP) precursor and an increase in cleaved PARP (at $24 \mathrm{kDa}$ ) were observed. Furthermore, decreases in caspase-3 and -9 precursors were also observed upon treatment with vorinostat and vorinostat-NPs. And, the level of cleaved caspase-3 was increased, as shown in Fig. 4B. Including the result of Bax expression, these results indicated that vorinostat-NPs induced apoptosis and affected the expression of apoptotic molecular signals to the same extent as did vorinostat. On treatment with vorinostat or vorinostat-NPs, actin was disrupted in HuCC-T1 cells as shown in Fig. 4C, indicating that vorinostat-NPs have a similar effect on apoptosis and protein changes at the cellular level as does vorinostat.

\section{In vivo antitumor activity of vorinostat-incorporated nanoparticles}

The increase in tumor volume and changes in body weight were monitored. As shown in Fig. 5a, the size of the tumor rapidly increased with time following treatment of empty-NPs. However, the volume of the tumor was significantly suppressed by treatment of vorinostat or vorinostat-NPs. Body weight did not significantly change with any of the treatments, indicating that neither vorinostat nor nanoparticles were significantly toxic to the mice, as shown in Fig. 5b. Interestingly, vorinostatNPs showed higher efficacy of tumor growth inhibition: tumor volume following treatment of vorinostat-NPs was almost $50 \%$ smaller than following treatment of vorinostat. Terminal deoxynucleotidyl transferase dUDT nick-end-labeling (TUNEL) staining of solid tumors supported these results, as shown in Fig. 5c: upon treatment of vorinostat-NPs, apoptosis was higher than treatment of vorinostat, whereas minimal apoptosis was seen upon treatment of empty-NPs. Interestingly, the expression of ac-histone $\mathrm{H} 3$ in tumor tissue was significantly increased upon treatment with vorinostat-NPs compared to emptyNPs and vorinostat as shown in Fig. 6. Furthermore, the expression levels of HDAC 1, 2, 3 and 4/5/7 were relatively decreased upon treatment of vorinostat and vorinostat-NPs, compared to treatment of empty-NPs. These results indicated that subcutaneous injection of vorinostat-NPs has similar or higher antitumor activity compared to vorinostat. 


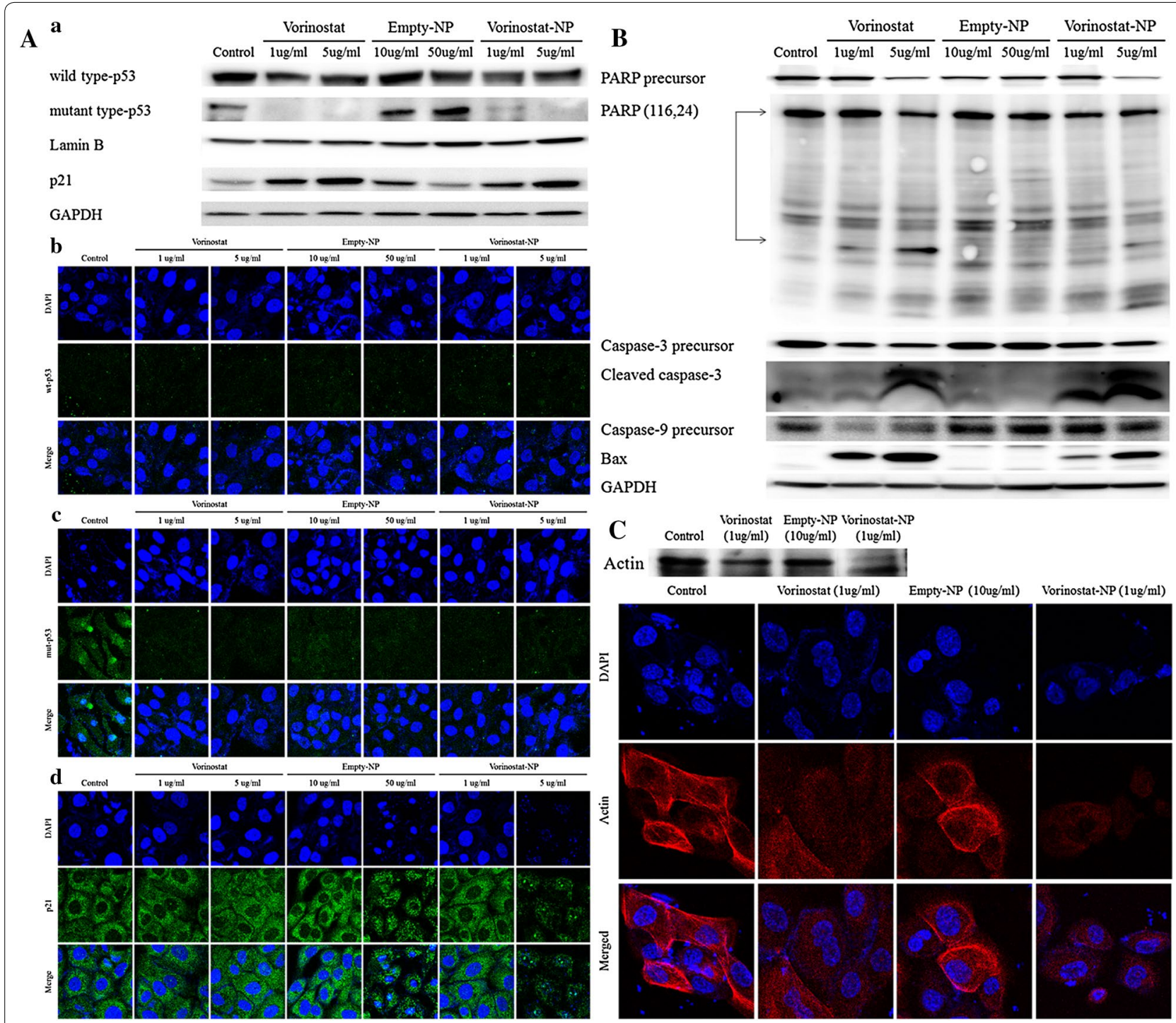

Fig. 4 Expression of signals after treatment with vorinostat and vorinostat-NPs. A $a$ Western blot analysis of apoptotic signal expression in HuCC-T1 cells following treatment with vorinostat or vorinostat-NPs. b-d Immunocytochemistry ( $\times 1200)$ of wild-type p53, mutant p53 and p21 of HuCCT1 cells after treatment with vorinostat or vorinostat-NPs. B Western blot analysis of PARP precursor, PARP, caspase-3 precursor, cleaved caspase-3, caspase-9 precursor and Bax. C Western blot analysis and immunocytochemistry of actin

To clarify the reason for the higher antitumor activity of the nanoparticles, near infrared (NIR)-dye-conjugated nanoparticles (NIR-NPs) were subcutaneously injected into a normal region as well as the site of the tumor, as shown in Fig. 7. Intact NIR-dye treated mice showed a rapid decrease in both the normal region and the tumor site. However, nanoparticle treated mice showed quite different results, i.e. nanoparticles remained longer at the tumor site than at the normal region. In particular, the strongest fluorescence intensity was observed at the center of the solid tumor at 1 day after treatment with NIR-NPs, whereas intact NIR-dye revealed the strongest fluorescence intensity in the region surrounding the solid tumor. The treatment of NIR-NPs revealed strong fluorescence intensity after 8 days for injection. These results confirmed that vorinostat-NPs have higher antitumor activity in vivo than does vorinostat.

\section{Discussion}

HDAC expression in cancer cells has a critical role in remodeling of chromatin structure, gene expression, cell cycle regulation and differentiation $[30,31]$. Increased HDAC activity is known to result in malignant tumor behavior [30]. In particular, Morine et al. reported that 

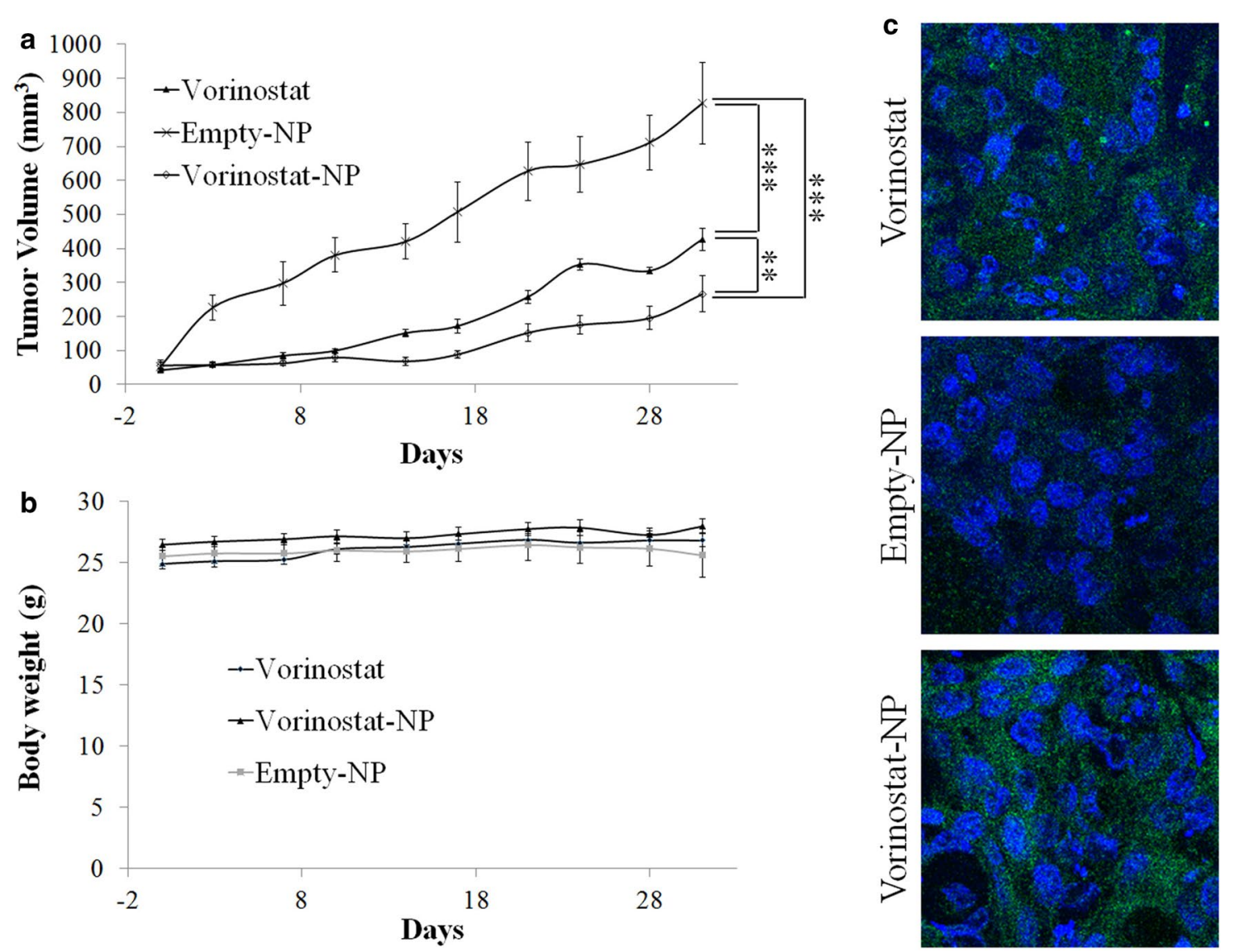

Fig. 5 Antitumor activity of vorinostat or vorinostat-NP in HuCC-T1 tumor xenograft mice model. (dose $50 \mathrm{mg}$ vorinostat $/ \mathrm{kg}$ ) a tumor volume; b body weight; c TUNEL assay $(\times 400)$ of extracted tumor tissues. HuCC-T1 human CCA cells $\left(1 \times 10^{7}\right)$ were implanted into the back of BALb/C nude mouse. 2 weeks later, vorinostat, empty-NPs or vorinostat-NPs were injected subcutaneously beside the solid tumor and the day of drug injection was set as day 0 . Tumor volume was calculated using the formula $V=\left(a \times[b]^{2}\right) / 2$, with $a$ being the largest and $b$ being the smallest diameter. For TUNNEL assay, tumors were isolated and fixed with $4 \%$ formamide after 30 days of injection

HDAC1 expression in intrahepatic CCA is significantly correlated with the stage of carcinogenesis and is related to malignant behaviors of cancer, such as angiogenesis, lymph node metastasis and vascular invasion [30]. They found that the survival rate of the HDAC1-positive group was significantly worse than that of the negative group. Furthermore, HDAC6 is also known to have a strong relationship with the stage of CCA and can thus be considered a clinic-pathological parameter for CCA [32]. Higher HDAC expression is associated with shorter survival times in gastric cancer patients and is regarded as an independent prognostic marker for gastric cancer [33]. Inhibition of the molecular action of HDAC using HDAC inhibitors is a promising candidate for cancer chemotherapy [34, 35].

HDAC inhibitors exert anticancer activities on human cancer cells through cell cycle arrest, growth arrest, activation of apoptotic pathways, autophagic cell death, reactive oxygen species (ROS)-mediated cell death and mitotic cell death etc. [35, 36]. HDAC inhibitors tightly bind to DNA histones and prevent the transcription/ expression of tumor suppressor genes by inducing histone acetylation [35, 36]. Several HDAC inhibitors which inhibits class I and II HDACs [36] show minimal intrinsic toxicity to human bodies but show dramatic anticancer efficacy for cancer [37]. In clinical trials, oral administration of vorinostat with promising anticancer activity was well tolerated in patients with GI cancers [3]. In animal tumor xenograft studies, intraperitoneal injection of vorinostat induced tumor necrosis and inhibited the growth of colon tumors through the inhibition of different subtypes of HDACs [5].

In our study, we fabricated vorinostat-NPs for treatment of CCA. As shown in Fig. 1, vorinostat-NPs have spherical shapes and small diameter $<100 \mathrm{~nm}$. They showed sustained drug release behavior over 5 days as shown in Fig. 1d. Especially, nanoparticles having higher vorinostat contents (vorinostat-NP20) showed slower 


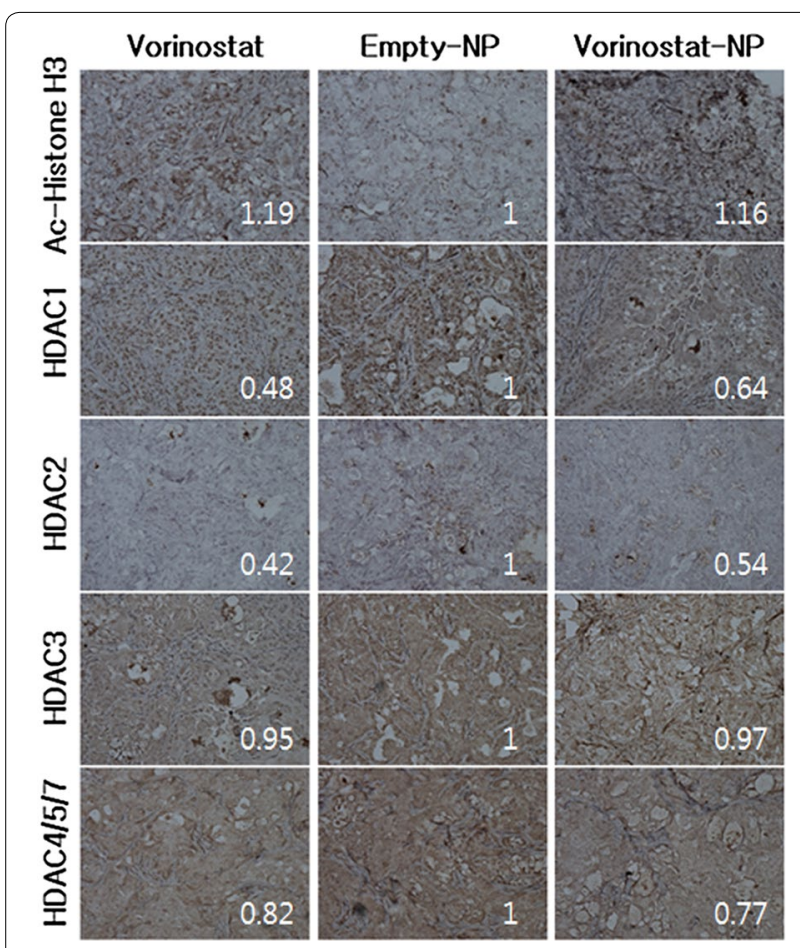

Fig. 6 Immunohistochemistry $(\times 400)$ of tumor tissues from HuCC-T1 cell bearing xenograft mouse model. To study HDAC expression, tumor tissues were stained with acetyl histone H3, HDAC1, HDAC2, HDAC3, and HDAC4/5/7 antibodies

release kinetic. These results might be due to that hydrophobic drug can be aggregated at higher drug loading contents and release of aggregated drug can be delayed compared to the nanoparticles with lower drug contents (vorinostat-NP10). These phenomena were frequently reported by several investigators. To assess biological activity, vorinostat-NPs were treated to $\mathrm{HuCC}-\mathrm{T} 1$ cells and their anticancer activity was compared to vorinostat itself at in vitro and in vivo. Vorinostat-NPs as well as vorinostat properly inhibited the growth of HuCC-T1 cells in vitro and the growth of tumor volume in vivo through inhibition of HDAC expression in the HuCCT1 cells and tumor tissues, as shown in Figs. 3, 5 and 6. Furthermore, vorinostat-NPs have higher antitumor activity compared to vorinostat, due to the sustained release properties of nanoparticles. The apoptotic signals of HuCC-T1 cells were also significantly altered upon treatment with vorinostat or vorinostat-NPs in vitro and in vivo as shown in Figs. 3, 4 and 5. As shown in Fig. 4, levels of mutant p53 were significantly decreased with little change in wild-type p53 and this suppression is correlated with the expression of PARP/cleaved caspase-3. Other researchers have reported that the expression of mutant p53 was significantly decreased in a dose- and time-dependent manner [38, 39]. For example, Yan et al.

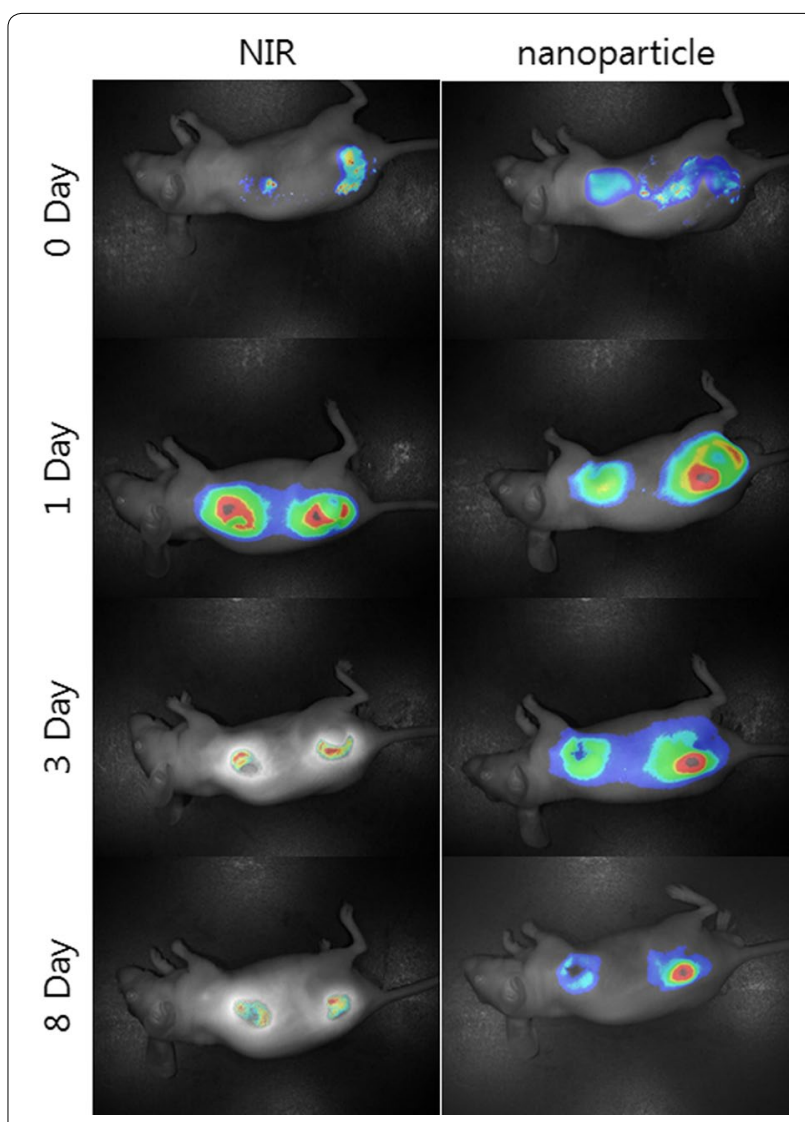

Fig. 7 In vivo fluorescence imaging of HuCC-T1 tumor xenograft mice model. NIR dye-incorporated NPs were simultaneously injected into a normal region and beside the tumor region of the back of mouse. Mouse were observed with the Maestro $2^{\mathrm{TM}}$ In Vivo imaging system at $780 \mathrm{~nm}$

found that disruption of HDAC8 expression significantly inhibits proliferation of cancer cells having mutant-type p53 irrespective of wild-type p53. In their results, colony formation of mutant-type p53 cell lines SW480 was remarkably decreased by treatment of vorinostat while wild-type p53 cell lines HCT116 showed little changes [40]. Furthermore, both vorinostat-NPs and vorinostat were able to arrest cell growth and induced apoptosis as shown in Figs. 3 and 4. And, they increased the expression of p21, a cyclin-dependent kinase inhibitor I, in a dose-dependent manner and this is correlated with other apoptosis signals. Thenaa et al. also reported that vorinostat inhibits mammary cell growth through altered p21 expression and cell cycle arrest [41]. Our results showed that vorinostat-incorporated nanoparticles as well as vorinostat itself also affects in induction of apoptotic signals, suppression of mutant-type p53, up-regulation of p21 and disruption of actin in HuCC-T1 cells. Furthermore, vorinostat-incorporated nanoparticles were also higher efficacy than vorinostat itself at in vivo animal 
tumor xenograft study. Treatment with vorinostat or vorinostat-incorporated nanoparticles increased acetylation of histone H3 (Ac-Histone H3) and then decreased HDAC expression as shown in Fig. 6. Di Gennaro et al. also reported that subcutaneous injection of vorinostat at a dose of $100 \mathrm{mg} / \mathrm{kg}$ against SW620 colorectal cancer xenografts increases Ac-Histone H3 and showed synergized effect with capecitabine in the inhibition of tumor growth [42]. Furthermore, intravenous injection of vorinostat or vorinostat-incorporated nanoparticles is known to sensitize radiotherapy and then effectively inhibit growth of PC3 tumor xenograft in mice [43].

Hydrogels or nanocarriers are known to improve antitumor activity of vorinostat in vivo animal tumor xenograft model [44-46]. Vorinostat-NPs caused antitumor activity, apoptotic expression in TUNEL assay and inhibitory activity of HDAC expression compared with vorinostat itself, as shown in Figs. 5 and 6. Antitumor activity of drugs can be improved at in vivo circumstances by use of biodegradable polymers for controlled drug release [41]. Therefore, these results can be explained by the sustained release properties of vorinostat-NPs. Li et al. reported that biodegradable thermosensitive hydrogel enhances the therapeutic efficacy of vorinostat and significantly inhibited intratumoral angiogenesis [44]. Furthermore, Mohamed et al. reported that polymeric micelles significantly enhance half-lives in blood and bioavailability of vorinostat in rats by intravenous injection and oral administration [45]. Nanocarriers also increase the half-life of vorinostat in blood and improve the antitumor activity of vorinostat [46]. Gref et al. reported potential of long blood circulation of core-shell type nanospheres composed of PLGA-PEG block copolymer rather than plain nanoparticles [28]. Systemic approach of chemotherapeutic agents is known to have limited clinical benefit due to the difficulties of drug delivery to CCA tumor [20, 21, 47]. For this reason, alternative treatment regimen is required to deliver the anticancer drugs to CCA tumor. Therefore, we focused on the possibility of drug delivery to CCA tumor by local administration of vorinostat-incorporated nanoparticles. Practically, growth of tumors originated in $\mathrm{HuCC}-\mathrm{T} 1$ cells in the back of the mice was effectively suppressed compared to vorinostat itself and empty nanoparticle treatment as shown in Fig. 5. To clarify vorinostat delivery to tumor tissues, NIR-dye was physically incorporated into the nanoparticles as similar to vorinostat and injected beside tumor tissues. As shown in Fig. 7, it is likely that nanoparticles were efficiently delivered to tumor tissues compared to free NIR-dye. Furthermore, NIR-NPs stayed longer in the tumor tissue than free NIR-dye. Practically, NIR-NPs were rapidly cleared from normal region but not in tumor region while free NIR-dye was rapidly cleared both in normal and tumor region. The reason of improved antitumor activity of vorinostat nanoparticles compared to vorinostat itself can be explained by these results. Other researcher also reported that nanoparticles can be stayed longer in the injection site and efficiently delivered to tumor tissues compared to free NIR-dye [48]. In other words, enhanced permeation and retention effect of macromolecules and nanomedicines in the tumor tissues also can be considered to explain these results $[49,50]$. In our results, vorinostat-NPs showed higher anticancer activity than intact vorinostat and have higher efficacy in the drug delivery to tumor tissue. We suggest that vorinostat nanoparticles are promising candidate to treat CCA.

\section{Conclusion}

We prepared vorinostat-NPs using biodegradable block copolymer for anticancer therapy in HuCC-T1 CCA cells. Vorinostat-NPs have similar anticancer activities in terms of growth inhibition, apoptosis and inhibition of HDAC expression in vitro to that of vorinostat alone. However, vorinostat-NPs show improved antitumor activity in xenograft mice model and a higher inhibition rate of HDAC expression in vivo. The higher anticancer activity of vorinostat-NPs can be explained by NIR-NPs, i.e. NIR-NPs were remained in the tumor tissue longer than did free NIR dye. We suggest that vorinostat nanoparticles can be used as a promising vehicle for HDAC-targeted chemotherapy in CCA cells.

\section{Methods \\ Materials}

Vorinostat was purchased from LC Labs. Co. (Woburn, MA, USA). LGE copolymer (Resomer ${ }^{\circledR}$ RGP d 50105) was purchased from Boehringer Ingelheim Pharma $\mathrm{GmbH}$ \& Co. (Ingelheim am Rhein, Germany). Pluronic F68, dimethyl sulfoxide (DMSO) and acetone were purchased from Sigma-Aldrich Chem. Co. (St. Louis, MO, USA). Dialysis membranes with molecular weight cutoffs of $8000 \mathrm{~g} / \mathrm{mol}$ were purchased from Spectra/PorTM (Spectrum Laboratories Inc, Rancho Dominguez, CA, USA). RPMI1640 media, fetal bovine serum (FBS) and all cell culture components were purchased from Life Technologies (Grand Island, NY, USA). All reagents and organic solvents used were of extra-pure grade.

\section{Fabrication of vorinostat-incorporated nanoparticles}

One hundred milligrams of LGE were dissolved in $10 \mathrm{ml}$ acetone. Ten and twenty mg of vorinostat was dissolved in 0.2 and $0.4 \mathrm{ml}$ of DMSO, respectively. Then, vorinostat solution was mixed with LGE/acetone solution. The mixed solution was dropped in $20 \mathrm{ml}$ of deionized 
water [Pluronic F68, $0.1 \%(\mathrm{w} / \mathrm{v})$ ] for $10 \mathrm{~min}$ and then the organic solvent was evaporated under vacuum. The nanoparticle solution was recovered by ultra-centrifugation at $100,000 \times g$ (Supra $30 \mathrm{~K}$, Vacuum High Speed Centrifuge, Hanil Science Industrial Co. Ltd., Incheon, Korea). Subsequently, harvested nanoparticles were washed with $10 \mathrm{ml}$ of deionized water and then harvested again by ultra-centrifugation. The washing procedure was repeated three times. The resulting nanoparticles were reconstituted in deionized water or lyophilized. To measure vorinostat content in the nanoparticles, $5 \mathrm{mg}$ of lyophilized nanoparticles were dissolved in DMSO. The drug content and loading efficiency of vorinostat in the vorinostat-NPs was evaluated using the Flexar high-performance liquid chromatography (HPLC) system (Perkin-Elmer Life and Analytical Sciences, Waltham, MA, USA).

Drug concentrations were determined using the HPLC system as follows: the Flexar HPLC system was equipped with a Solvent Manager 5-CH degasser, an autosampler, a quaternary LC pump, a column oven and an UV/VIS detector. Chromatography was performed on a guard column (SecurityGuard ${ }^{\circledR}$ Guard Cartridge Kit; Phenomenex, Torrance, CA, USA) and a C18 column (Brownlee $\mathrm{C} 18^{\circledR}, 5$ micrometer, $150 \times 4.6$; Perkin Elmer) at $37^{\circ} \mathrm{C}$. Vorinostat was eluted isocratically with mobile phase (acetonitrile/0.1\% formic acid at a ratio of 22/78) at a flow rate of $1 \mathrm{ml} / \mathrm{min}$ and monitored at $241 \mathrm{~nm}$. Chromatograms were recorded and integrated with the Chromera 2.1 system software (Perkin Elmer Life and Analytical Sciences, Waltham, MA, USA).

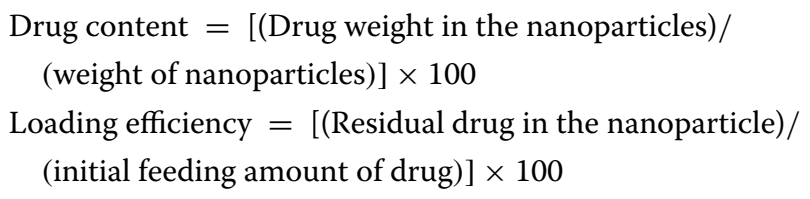

Characterization of vorinostat-incorporated nanoparticles The morphology of the nanoparticles was observed using TEM (JEM-2000 FX II microscope, JEOL, Tokyo, Japan). The nanoparticle solution was dropped onto a carbon film coated on a copper grid and then the nanoparticles were negatively stained with phosphotungstic acid $(0.05 \% \mathrm{w} / \mathrm{w})$. TEM observation was performed at an accelerating voltage of $80 \mathrm{kV}$. Particle size was measured using the Nano-ZS apparatus (Malvern Instruments, Malvern, UK). Nanoparticles were reconstituted in deionized water (nanoparticle concentration $0.1 \mathrm{mg} /$ $\mathrm{ml}$ ) and then used to determine particle size. The crystallinity of vorinostat and vorinostat-NPs were analyzed using XRD (Rigaku D/Max-1200, Rigaku, Tokyo, Japan) equipped with $\mathrm{Ni}$-filtered $\mathrm{Cu}$ Ka radiation (40 kV,
$20 \mathrm{~mA}$ ). The vorinostat powder and lyophilized nanoparticle solid were used to measure crystallinity using XRD.

\section{Drug release study}

Drug release testing was performed using phosphatebuffered saline (PBS; $10 \mathrm{mM}, \mathrm{pH} 7.4$ ) solution at $37{ }^{\circ} \mathrm{C}$. Five milligrams of nanoparticles in $1 \mathrm{ml}$ of deionized water were added to $4 \mathrm{ml}$ of PBS and this solution was then introduced into a dialysis tube. This dialysis tube was immersed in a $100 \mathrm{ml}$ bottle with $95 \mathrm{ml}$ of PBS. Whole media were taken at predetermined time intervals and exchanged with fresh PBS. The concentration of the released drug was measured using the HPLC system. The percentage of released drug was calculated from following equation: [(amount of released drug/total weight of drug in the nanoparticles) $\times 100]$.

\section{Cell culture}

HuCC-T1 cell line was obtained from the Health Science Research Resources Bank (Osaka, Japan) and maintained with RPMI1640 medium supplemented with $10 \%$ heatinactivated FBS and $1 \%$ penicillin/streptomycin at $37{ }^{\circ} \mathrm{C}$ in a humidified atmosphere containing $5 \% \mathrm{CO}_{2}$.

\section{Cell cytotoxicity and growth inhibition study}

HuCC-T1 cells were seeded in 24-well plates at a density of $3 \times 10^{4}$ and $3 \times 10^{5}$ cells per well for the growth inhibition and cytotoxicity assays, respectively. Following this, each plate was incubated overnight in a $\mathrm{CO}_{2}$ incubator. Vorinostat in DMSO and vorinostat-NPs were diluted with RPMI1640 medium containing 10 \% FBS for the growth inhibition assay at various concentrations and then added to HuCC-T1 cells in 24-well plates following $24 \mathrm{~h}$ incubation. The cytotoxicity assay was carried out using serumfree RPMI1640 media. The control was treated with $0.1 \%$ (v/v) DMSO. Cells were trypsinized, harvested and resuspended in PBS. Trypan blue was added and the number of cells was counted using the Countess ${ }^{\mathrm{TM}}$ Automated Cell Counter (Invitrogen, Carlsbad, CA, USA). The reduction of viable cells by treatment of vorinostat or vorinostatincorporated nanoparticles compared to control treatment was calculated and expressed as mean $\pm \mathrm{SD}$.

\section{Apoptosis and necrosis analysis}

HuCC-T1 cells were seeded in 6-well plates at a density of $1 \times 10^{6}$ cells per well and exposed to various concentrations of vorinostat and vorinostat-NPs for $24 \mathrm{~h}$. The cells were harvested, washed with PBS, resuspended in $500 \mu \mathrm{l}$ binding buffer and stained with FITC-conjugated Annexin $\mathrm{V}$ for apoptosis analysis and with PI for necrosis analysis. These cells were analyzed by flow cytometry (BD biosciences, San Jose, CA, USA). 


\section{Western blot analysis and immunocytochemistry}

HuCC-T1 cells were seeded in 6-well plates at a density of $1 \times 10^{6}$ cells per well and exposed to various concentrations of vorinostat and vorinostat-NPs for $24 \mathrm{~h}$. Cells were trypsinized and washed with cold PBS. The cells were collected by centrifugation and lysed in lysis buffer containing protease inhibitors [50 mM Tris, $150 \mathrm{mM}$ $\mathrm{NaCl}, 1 \% \mathrm{NP}-40,0.5 \%$ deoxycholic acid, 0.1 \% sodium dodecyl sulfate (SDS)] with phenylmethylsulfonyl fluoride and a protease inhibitor cocktail (Roche Diagnostics, Basel, Switzerland). The cell suspension was cleared by centrifugation at $14,000 \times g$ for $30 \mathrm{~min}$ at $4{ }^{\circ} \mathrm{C}$ and then supernatant or cell lysates were collected. The protein concentration was determined using the BCA Protein Assay kit (Pierce, Rockford, IL, USA).

For western blotting, $50 \mu \mathrm{g}$ protein was subjected to SDSpolyacrylamide gel electrophoresis (SDS-PAGE), transferred to a polyvinyl difluoride membrane, blocked with $5 \%$ skim milk in TBS-T and probed with an appropriate primary antibody followed by a secondary HRP-conjugated antibody. Proteins were detected by chemiluminescence. Proteins were quantified by digital analyses.

\section{Antitumor activity of vorinostat-incorporated nanoparticles against the animal tumor xenograft model}

To assess the antitumor activity of vorinostat-NPs, a tumor xenograft model was prepared by subcutaneous injection of HuCC-T1 cells into the backs of nude mice. HuCC-T1 cells $\left(1 \times 10^{7}\right.$ cells $)$ in a total volume of $100 \mu \mathrm{l}$ were subcutaneously injected into the backs of male nude mice (5-week-old and 20-25 g in weight; Orient, Seongnam, South Korea). When the solid tumor reached approximately $4-5 \mathrm{~mm}$ in diameter, emptyNPs, vorinostat and vorinostat-NPs were injected subcutaneously adjacent to the solid tumor. Treatment dose was adjusted to $1 \mathrm{mg}$ vorinostat $(50 \mathrm{mg} / \mathrm{kg}$ ). A total of 18 mice were divided into three groups, as follows: (1) vorinostat-injected, (2) empty-NPs injected and (3) vorinostat-NP injected. Body weight and tumor volume were measured twice a week, starting on the first day of treatment. Two perpendicular diameters of the tumor were measured and tumor volume was calculated using the formula $V=\left(a \times[b]^{2}\right) / 2$, with $a$ being the largest and $b$ being the smallest diameter. The animal study was carried out according to the guidelines of the Animal Treatment and Research Council of Pusan National University.

\section{Immunohistochemistry}

After 30 days of injection, tumors were isolated and fixed in $4 \%$ formamide, paraffin-embedded and sliced for hematoxylin and eosin (H\&E) staining or for the TUNEL assay. For immunohistochemical staining of the tumors, acetyl histone $\mathrm{H} 3$ antibody was diluted to 1:500 and HDAC1 antibody was dilued to 1:100. HDAC2, HDAC3, and HDAC4/5/7 antibodies were diluted to 1:200. Staining was performed using an Envision kit (Life Technologies, Carlsbad, CA, USA) according to the manufacturer's protocol.

\section{Fluorescence imaging of solid tumor-bearing mice}

To study biodistribution of vorinostat-NPs, hydrophobic NIR-dye (XenoLight DiR, Caliper Lifesciences, MA 01748-1668, USA) was incorporated into the nanoparticles. Four milligrams of hydrophobic NIR-dye was dissolved in $0.2 \mathrm{ml}$ of DMSO and mixed with $100 \mathrm{mg}$ of LGE dissolved in acetone. This solution was dropped in $20 \mathrm{ml}$ of deionized water [Pluronic F68, $0.1 \%(\mathrm{w} / \mathrm{v})$ ] for $10 \mathrm{~min}$ and then organic solvent was evaporated under vacuum. After that, hydrophobic NIR-NPs were harvested by same procedure as described above. To measure content of hydrophobic NIR-dye in the nanoparticles, hydrophobic NIR-NPs were dissolved in $10 \mathrm{ml}$ of DMSO and the concentration was measured with fluorescence spectrophotometer (RF-5301 fluorescence spectrofluorophotometer, Shimadzu, Tokyo, Japan). The content of hydrophobic near-infrared was calculated to $3.6 \%(\mathrm{w} / \mathrm{w})$.

For tumor imaging, NIR-NPs $(50 \mathrm{mg} / \mathrm{kg}$ ) were injected subcutaneously beside tumor tissue. To compare uptake of nanoparticles at tumor tissue and normal tissue, same quantity of NIR-NPs was also injected subcutaneously in the normal region. Mouse was observed using the Maestro $2^{\mathrm{TM}}$ In Vivo imaging system (Cambridge Research and Instruments, Inc., Woburn, MA 01801, USA) at $780 \mathrm{~nm}$.

\section{Quantification of image intensity}

Quantification of staining intensities was calculated using the ImageJ (ver 1.42q) software (NIH, Bethesda, MD, USA).

\section{Statistical analysis}

Statistical analyses of the data from treated and untreated cells were performed using the Student's $t$ test. A p value $<0.05$ was considered to be statistically significant.

\section{Abbreviations}

vorinostat-NPs: vorinostat-incorporated nanoparticles; HDAC: histone deacetylase; HDACi: histone deacetylase inhibitor; Gl: gastrointestinal; CCA: cholangiocarcinoma; LGE: poly(DL-lactide-co-glycolide)-b-poly(ethylene glycol); DMSO: dimethyl sulfoxide; FBS: fetal bovine serum; TEM: transmission electron microscopy; XRD: X-ray powder diffractograms; PARP: poly-ADP ribose polymerase; SDS: sodium dodecyl sulfate; PAGE: polyacrylamide gel electrophoresis; TUNEL: terminal deoxynucleotidyl transferase dUDP nick-end-labeling; NIR: near-infrared; NIR-NPs: NIR-dye conjugated nanoparticles; empty-NPs: empty nanoparticles.

\section{Authors' contributions}

TWK carried out fabrication of vorinostat-loaded nanoparticles and cell culture study. DHK carried out animal study using mouse tumor xenograft model. YIJ conceived of this research and participated in its design. DHK drafted a manuscript and organized all the procedures. All authors read and approved the final manuscript. 


\section{Author details}

${ }^{1}$ Biomedical Research Institute, Pusan National University Hospital, 179 Gudeok-ro, Seo-gu, Busan 602-739, Republic of Korea. ${ }^{2}$ School of Medicine Pusan National University, Yangsan, Gyeongnam 626-770, Republic of Korea. ${ }^{3}$ Department of Internal Medicine, Pusan National University Yangsan Hospital, Yangsan, Gyeongnam 626-770, Republic of Korea.

\section{Acknowledgements}

This study was supported by a grant of the Korean Health Technology R\&D Project, Ministry of Health and Welfare, Republic of Korea (Project No. HI14C2220).

\section{Compliance with ethical guidelines}

\section{Competing interests}

The authors declare that they have no competing interests.

Received: 6 April 2015 Accepted: 10 September 2015

Published online: 26 September 2015

\section{References}

1. Richon VM. Cancer biology: mechanism of antitumour action of vorinostat (suberoylanilidehydroxamic acid), a novel histone deacetylase inhibitor. Br J Cancer. 2006;95:S2-6.

2. Takada Y, Gillenwater A, Ichikawa H, Aggarwal BB. Suberoylanilidehydroxamic acid potentiates apoptosis, inhibits invasion, and abolishes osteoclastogenesis by suppressing nuclear factor-kappaB activation. J Biol Chem. 2006:281:5612-22.

3. Doi T, Hamaguchi T, Shirao K, Chin K, Hatake K, Noguchi K, et al. Evaluation of safety, pharmacokinetics, and efficacy of vorinostat, a histone deacetylase inhibitor, in the treatment of gastrointestinal (Gl) cancer in a phase I clinical trial. Int J Clin Oncol. 2013;18:87-95.

4. Claerhout S, Lim JY, Choi W, Park YY, Kim K, Kim SB, et al. Gene expression signature analysis identifies vorinostat as a candidate therapy for gastric cancer. PLoS One. 2011;6:e24662.

5. Jin JS, Tsao TY, Sun PC, Yu CP, Tzao C. SAHA inhibits the growth of colon tumors by decreasing histone deacetylase and the expression of cyclin D1 and survivin. Pathol Oncol Res. 2012;18:713-20.

6. Chinnaiyan P, Chowdhary S, Potthast L, Prabhu A, Tsai YY, Sarcar B, et al. Phase I trial of vorinostat combined with bevacizumab and CPT-11 in recurrent glioblastoma. Neuro Oncol. 2012;14:93-100.

7. Ramaswamy B, Fiskus W, Cohen B, Pellegrino C, Hershman DL, Chuang E, et al. Phase I-II study of vorinostat plus paclitaxel and bevacizumab in metastatic breast cancer: evidence for vorinostat-induced tubulin acetylation and Hsp90 inhibition in vivo. Breast Cancer Res Treat. 2012;132:1063-72.

8. Liu YL, Yang PM, Shun CT, Wu MS, Weng JR, Chen CC. Autophagy potentiates the anti-cancer effects of the histone deacetylase inhibitors in hepatocellular carcinoma. Autophagy. 2010;6:1057-65.

9. KwakTW, Kim do H, Chung CW, Lee HM, Kim CH, Jeong Yl, et al. Synergistic anticancer effects of vorinostat and epigallocatechin-3-gallate against HuCC-T1 human cholangiocarcinoma cells. Evid Based Complement Altern Med. 2013;2013:185158.

10. Lim JH. Cholangiocarcinoma: morphologic classification according to growth pattern and imaging findings. Am J Roentgenol. 2003;181:819-27.

11. Khan SA, Taylor-Robinson SD, Toledano MB, Beck A, Elliott P, Thomas HC. Changing international trends in mortality rates for liver, biliary and pancreatic tumours. J Hepatol. 2002;37:806-13.

12. Sirica AE. Cholangiocarcinoma: molecular targeting strategies for chemoprevention and therapy. Hepatology. 2005;41:5-15.

13. Ciombor KK, Goff LW. Advances in the management of biliary tract cancers. Clin Adv Hematol Oncol. 2013;11:28-34.

14. Brunner TB, Eccles CL. Radiotherapy and chemotherapy as therapeutic strategies in extrahepatic biliary duct carcinoma. Strahlenther Onkol. 2010;186:672-80.

15. Skipworth JR, Olde Damink SW, Imber C, Bridgewater J, Pereira SP, Malagó M. Surgical, neo-adjuvant and adjuvant management strategies in biliary tract cancer. Aliment Pharmacol Ther. 2011;34:1063-78.
16. Rizvi S, Gores GJ. Pathogenesis, diagnosis and management of cholangiocarcinoma. Gastroenterology. 2013;145:1215-29.

17. Cereda S, Passoni P, Reni M, Viganò MG, Aldrighetti L, Nicoletti R, et al. The cisplatin, epirubicin, 5-fluorouracil, gemcitabine (PEFG) regimen in advanced biliary tract adenocarcinoma. Cancer. 2010;116:2208-14.

18. Kim ST, Park JO, Lee J, Lee KT, Lee JK, Choi SH, et al. A phase II study of gemcitabine and cisplatin in advanced biliary tract cancer. Cancer. 2006;106:1339-46.

19. Valle J, Wasan H, Palmer DH, Cunningham D, Anthoney A, Maraveyas $A$, et al. Cisplatin plus gemcitabine versus gemcitabine for biliary tract cancer. N Engl J Med. 2010;362:1273-81.

20. Leong E, Chen WW, Ng E, Van Hazel G, Mitchell A, Spry N. Outcomes from combined chemoradiotherapy in unresectable and locally advanced resected cholangiocarcinoma. J Gastrointest Cancer. 2012;43:50-5.

21. Furuse J, Okusaka T. Targeted therapy for biliary tract cancer. Cancers (Basel). 2011;3:2243-54.

22. Braga HJ, Imam K, Bluemke DA. MR imaging of intrahepatic cholangiocarcinoma: use of ferumoxides for lesion localization and extension. Am J Roentgenol. 2011;177:111-4.

23. Lee Y, Lee JS, Kim CM, Jeong JY, Choi JI, Kim MJ. Area of paradoxical signal drop after the administration of superparamagnetic iron oxide on the T2-weighted image of a patient with lymphangitic metastasis of the liver. Magn Reson Imaging. 2008;26:577-82.

24. Tang T, Zheng JW, Chen B, Li H, Li X, Xue KY, et al. Effects of targeting magnetic drug nanoparticles on human cholangiocarcinoma xenografts in nude mice. Hepatobiliary Pancreat Dis Int. 2007;6:303-7.

25. Towata T, Komizu Y, Kariya R, Suzu S, Matsumoto Y, Kobayashi N, et al. Hybrid liposomes inhibit the growth of cholangiocarcinoma by induction of cell cycle arrest in G1 phase. Bioorg Med Chem Lett. 2010;20:3680-2.

26. Chung KD, Jeong Yl, Chung CW, Kim do H, Kang DH. Anti-tumor activity of all-trans retinoic acid-incorporated glycol chitosan nanoparticles against HuCC-T1 human cholangiocarcinoma cells. Int J Pharm. 2012;422:454-61.

27. Hwang JH, Choi CW, Kim do HW, Kim H, KwakTW, Lee HM, et al. Dextranb-poly(L-histidine) copolymer nanoparticles for $\mathrm{pH}$-responsive drug delivery to tumor cells. Int J Nanomedicine. 2013;8:3197-207.

28. Gref R, Minamitake Y, Peracchia MT, Trubetskoy V, Torchilin V, Langer R. Biodegradable long-circulating polymeric nanospheres. Science. 1994;263:1600-3.

29. Oh JK. Polylactide (PLA)-based amphiphilic block copolymers: synthesis, self-assembly and biomedical applications. Soft Matter. 2011;7:5096-108.

30. Morine Y, Shimada M, Iwahashi S, Utsunomiya T, Imura S, Ikemoto T, et al. Role of histone deacetylase expression in intrahepatic cholangiocarcinoma. Surgery. 2012;151:412-9.

31. Sun WJ, Zhou X, Zheng JH, Lu MD, Nie JY, Yang XJ, et al. Histone acetyltransferases and deacetylases: molecular and clinical implications to gastrointestinal carcinogenesis. Acta Biochim Biophys Sin (Shanghai). 2012;44:80-91.

32. Boonjaraspinyo S, Boonmars T, Kaewkes S, Laummaunwai P, Pinlaor S, Loilome W, et al. Down-regulated expression of HSP70 in correlation with clinicopathology of cholangiocarcinoma. Pathol Oncol Res. 2012;18:227-37

33. Weichert W, Röske A, Gekeler V, Beckers T, Ebert MP, Pross M, et al. Association of patterns of class I histone deacetylase expression with patient prognosis in gastric cancer: a retrospective analysis. Lancet Oncol. 2008;9:139-48.

34. Weichert W. HDAC expression and clinical prognosis in human malignancies. Cancer Lett. 2009;280:168-76.

35. Fang JY. Histone deacetylase inhibitors, anticancerous mechanism and therapy for gastrointestinal cancers. J Gastroenterol Hepatol. 2005;20:988-94.

36. Xu WS, Parmigiani RB, Marks PA. Histone deacetylase inhibitors: molecular mechanisms of action. Oncogene. 2007;26:5541-52.

37. Vigushin DM, Coombes RC. Histone deacetylase inhibitors in cancer treatment. Anticancer Drugs. 2012;13:1-13.

38. Yan W, Liu S, Xu E, Zhang J, Zhang Y, Chen X, et al. Histone deacetylase inhibitors suppress mutant p53 transcription via histone deacetylase 8. Oncogene. 2013;32:599-609.

39. Li D, Marchenko ND, Moll UM. SAHA shows preferential cytotoxicity in mutant p53 cancer cells by destabilizing mutant p53 through inhibition of the HDAC6-Hsp90 chaperone axis. Cell Death Differ. 2011;18:1904-13. 
40. Chen YX, Fang JY, Zhu HY, Lu R, Cheng ZH, Qiu DK. Histone acetylation regulates p21WAF1 expression in human colon cancer cell lines. World $J$ Gastroenterol. 2004;10:2643-6.

41. Said TK, Moraes RCB, Sinha R, Medina D. Mechanisms of suberoylanilide hydroxamic acid inhibition of mammary cell growth. Breast Cancer Res. 2001:3:122-33.

42. Di Gennaro E, Piro G, Chianese MI, Franco R, Di Cintio A, Moccia T, Luciano A, de Ruggiero I, Bruzzese F, Avallone A, Arra C, Budillon A. Vorinostat synergises with capecitabine through upregulation of thymidine phosphorylase. Br J Cancer. 2010;103:1680-91.

43. Wang EC, Min Y, Palm RC, Fiordalisi JJ, Wagner KT, Hyder N, Cox AD, Caster JM, Tian X, Wang AZ. Nanoparticle formulations of histone deacetylase inhibitors for effective chemoradiotherapy in solid tumors. Biomaterials. 2015;51:208-15.

44. Li J, Gong C, Feng X, Zhou X, Xu X, Xie L, et al. Biodegradable thermosensitive hydrogel for SAHA and DDP delivery: therapeutic effects on oral squamous cell carcinoma xenografts. PLoS One. 2012;7:e33860.

45. Mohamed EA, Zhao Y, Meshali MM, Remsberg CM, Borg TM, Foda $A M$, et al. Vorinostat with sustained exposure and high solubility in poly(ethylene glycol)-b-poly(DL-lactic acid) micelle nanocarriers: characterization and effects on pharmacokinetics in rat serum and urine. J Pharm Sci. 2012;101:3787-98.
46. Kim JY, Shim G, Choi HW, Park J, Chung SW, Kim S, et al. Tumor vasculature targeting following co-delivery of heparin-taurocholate conjugate and suberoylanilide hydroxamic acid using cationic nanolipoplex. Biomaterials. 2012;33:4424-30.

47. Thomas MB. Systemic and targeted therapy for biliary tract tumors and primary liver tumors. Surg Oncol Clin N Am. 2014;23:369-81.

48. Park W, Park SJ, Na K. The controlled photoactivity of nanoparticles derived from ionic interactions between a water soluble polymeric photosensitizer and polysaccharide quencher. Biomaterials. 2011;32:8261-70.

49. Maeda H, Wu J, Sawa T, Matsumura Y, Hori K. Tumor vascular permeability and the EPR effect in macromolecular therapeutics: a review. J Control Release. 2000;65:271-84.

50. Iyer AK, Khaled G, Fang J, Maeda H. Exploiting the enhanced permeability and retention effect for tumor targeting. Drug Discov Today. 2006:11:812-8.

\section{Submit your next manuscript to BioMed Central and take full advantage of:}

- Convenient online submission

- Thorough peer review

- No space constraints or color figure charges

- Immediate publication on acceptance

- Inclusion in PubMed, CAS, Scopus and Google Scholar

- Research which is freely available for redistribution

Submit your manuscript at 